\title{
Relationship between dielectric properties and critical behavior of the electric birefringence in binary liquid mixtures
}

\author{
Sylwester J. Rzoska, ${ }^{*}$ Vittorio Degiorgio, and Mario Giardini \\ Dipartimento di Elettronica, Università di Pavia, via Abbiategrasso 209, 27100 Pavia, Italy
}

(Received 21 January 1994)

\begin{abstract}
We present experimental results on the critical exponent $\psi_{\mathrm{EKE}}$ describing the divergence of the Kerr constant of binary liquid mixtures near the critical consolute point. We show that the measured value of $\psi_{\mathrm{EKE}}$ agrees with the theoretical prediction only if the measurement is performed with a mixture of two liquids presenting a small mismatch in the dielectric constant, and that the measured $\psi_{\text {EKE }}$ grows as the dielectric constant mismatch increases. Such findings are consistent with a recent model which assumes that the elongation of critical fluctations along the direction of the electric field can become so strong that fluctuations in the direction perpendicular to the electric field may cross over from Ising to meanfield behavior.
\end{abstract}

PACS number(s): 64.70.Ja, 64.60.Ht, 78.20.Fm

\section{INTRODUCTION}

In the last few years several groups have studied effects due to the application of a static electric field $E$ in the vicinity of the critical consolute point of binary liquid mixtures. Two different effects have been experimentally investigated: the electrooptic Kerr effect (EKE) [1-9], and the nonlinear dielectric effect (NDE) [10-12]. In the former case, one measures the Kerr constant $B$, defined as

$$
B=\frac{1}{\lambda} \frac{\Delta n}{E^{2}}=\frac{1}{\lambda} \frac{n_{\|}-n_{\perp}}{E^{2}},
$$

where $\Delta n=n_{\|}-n_{\perp}$ is the anisotropy in refractive index due to the field $E$, and $\lambda$ is the wavelength of the light beam which is used to probe the induced birefringence. In the latter case, the experiment gives the quantity

$$
D=\frac{\Delta \varepsilon}{E^{2}}=\frac{\varepsilon_{\|}^{E}-\varepsilon}{E^{2}},
$$

where $\varepsilon_{\|}^{E}$ denotes the dielectric constant component parallel to the applied field, and $\varepsilon$ is the dielectric constant in the limit of zero static electric field. The dielectric constants are measured at radio frequencies.

Under the assumption that $E$ is sufficiently small, both $B$ and $D$ take values which are independent of $E$ and are determined only by the equilibrium properties of the system.

Both quantities $B$ and $D$ exhibit a marked increase on approaching the critical consolute point. Theoretical models [13-15] predict a power-law divergence of $D$ and $B$ as functions of the reduced temperature $t=\left|T-T_{c}\right| / T_{c}, T_{c}$ being the critical temperature:

$$
B_{c}, D_{c}=A_{B, D} t^{-\psi} \text {, }
$$

\footnotetext{
"Present address: Institute of Physics, Silesian University, ul. Uniwersytecka 4, 40-007 Katowice, Poland.
}

where the index $c$ denotes the critical part of the given effect, $A_{B}$ and $A_{D}$ are critical amplitudes, and the critical exponent is given by

$$
\psi=d v-2 \gamma \cong 3(0.63)-2(1.24)=0.59,
$$

where $d$ is the dimensionality of the space, and $v$ and $\gamma$ are the critical exponents for the correlation length and osmotic compressibility, respectively.

However, the available experimental results give values of $\psi_{\mathrm{EKE}}$ in the range $0.60-0.88[1-8]$ and of $\psi_{\mathrm{NDE}}$ around 0.4 [10-12]. The value of the critical exponent is the only, but fundamental, point of disagreement between theory and experiments. A possible explanation of the disagreement was proposed in Ref. [16] by assuming that the elongation of critical fluctuations along the direction of $E$ can become so strong that fluctuations in the direction perpendicular to $E$ may crossover from Ising to mean-field behaviors. This would yield the following critical exponents: $\psi_{\mathrm{NDE}} \approx 0.37$ and $\psi_{\mathrm{EKE}}=0.85$. In principle, if the model is correct, one should be able to see the crossover from $\psi_{\mathrm{EKE}}=0.59$ to $\psi_{\mathrm{EKE}}=0.85$ with a specific binary mixture by simply increasing the value of $E$. In practice, as discussed below, it appears to be easier to change the magnitude of the induced anisotropy, at fixed reduced temperature and fixed field, by acting on the dielectric constant mismatch between the two components of the liquid mixture.

In this article we present experimental results for the electro-optic Kerr effect. We show that the measured value of $\psi_{\mathrm{EKE}}$ agrees with the theoretical prediction only if the measurement is performed with a mixture of two liquids presenting a small mismatch in the dielectric constants, and that the measured $\psi_{\text {EKE }}$ grows as the dielectric constant mismatch increases. All available data indicate the existence of a clear correlation between the measured $\psi_{\text {EKE }}$ and the quantity $\left(\varepsilon_{1}-\varepsilon_{2}\right)^{2} / \varepsilon$, where $\varepsilon_{1}$ and $\varepsilon_{2}$ are the dielectric constants of the two separate components of the liquid mixture, and $\varepsilon$ is the dielectric constant of the mixture. Such findings are consistent with the model discussed in Ref. [16]. 


\section{EXPERIMENTAL RESULTS}

A general description of electric birefringence can be found in Ref [17]. Our measurement of the Kerr constant as a function of the reduced temperature was performed with an apparatus similar to that used in previous experiments [5-8]. We have investigated three binary mixtures, all possessing an upper consolute point: (a) (士)-1-phenylethanol and dodecane, (b) 2-phenylethanol and decane, and (c) $n$-butylbenzene and propylene carbonate. The three mixtures present very different values for the mismatch in dielectric constant: (a) $\varepsilon_{1}-\varepsilon_{2} \cong 5$, (b) $\varepsilon_{1}-\varepsilon_{2} \cong 10$, and (c) $\varepsilon_{1}-\varepsilon_{2} \simeq 50$. The critical temperature $T_{c}$ and the critical mass fraction of the first component $x_{c}$ were established by the visual method. The obtained values are (a) $T_{c}=21.6^{\circ} \mathrm{C}, x_{c}=0.545$; (b) $T_{c}=44.9^{\circ} \mathrm{C}$, $x_{c}=0.51$, and (c) $T_{c}=18.9^{\circ} \mathrm{C}, x_{c}=0.41$.

The sensitivity of EKE and NDE to molecular properties of liquids makes the estimation of the noncritical background effect rather difficult. In this work the noncritical background contribution was determined by the method employed in previous NDE [18] and EKE [4] studies. By exploiting the fact that in the three investigated solutions-( \pm -1-phenylethanol and dodecane, 2phenylethanol and decane, and $n$-butylbenzene and propylene carbonate - the background is due mainly to the first component, the background contribution was obtained from electric birefringence measurements performed with reference solutions containing carbon tetrachloride as the second component, each prepared with the same volume fraction of Kerr-active component as in the critical solution. The choice of carbon tetrachloride is motivated by the fact that by itself it presents a negligible Kerr effect, and, at the same time, the reference solutions used are not close to phase separation points.

In Fig. 1 we show the critical Kerr constant measurements, performed as function of the reduced temperature, in mixtures (a) and (c). The straight lines represent a fit with the power-law behavior predicted by Eq. (3). The best-fit exponents are $\psi_{\mathrm{EKE}}=0.58 \pm 0.04$ for system (a), and $\psi_{\mathrm{EKE}}=0.83 \pm 0.04$ for system (c). For the case of system (b), we show in Fig. 2 the measured values of the total Kerr constant. An interesting feature of this solution is the negative sign of the noncritical contribution, due to the fact that the permanent dipole moment is not parallel to the optical polarizability vector in the 2-phenylethanol molecule. The full line through the experimental points is the best-fit curve describing the superposition of critical and background effects. The obtained value of the critical exponent is $\psi_{\mathrm{EKE}}=0.64 \pm 0.04$.

\section{DISCUSSION}

On approaching the critical consolute point of a binary liquid mixture, large-scale fluctuations appear. Critical fluctuations can be described as isotropic droplets which can be anisotropically deformed by an external field. If the two components present a mismatch in the dielectric constant, then the deformation (elongation) can be induced by the electric field $[17,19]$. Convincing experimental evidence of such a deformation is given by recent

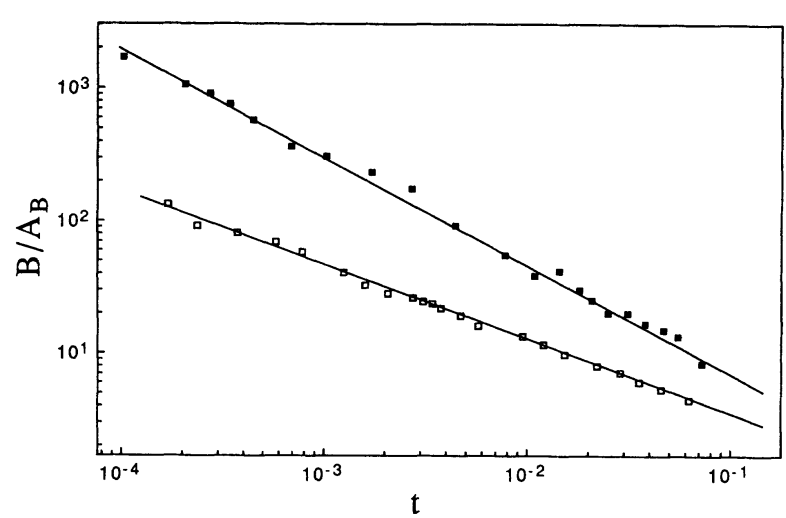

FIG. 1. The critical part of the Kerr constant of the liquid mixtures ( \pm )-1-phenylethanol and dodecane (open squares), and propylene carbonate and $n$-butylbenzene (full squares), measured as a function of the reduced temperature $t=\left(T-T_{c}\right) / T_{c}$.

studies of the anisotropy of the structure factor in a critical solution in presence of a static electric field using small-angle light scattering [9].

The situation described in this paper bears some similarity to studies of critical solutions under shear flow, where the elongation of critical fluctuations due to the action of the velocity gradient influences the critical properties [20]. It has indeed been shown that, in the regime of low shear rates $S \tau<1$ ( $S$ and $\tau$ are shear rate and relaxation time, respectively), the results are described by the Ising values of the critical exponents $(\gamma \approx 1.24$ and $\beta \approx 0.33$, respectively); whereas, for $S \tau>1$, mean-field exponents are found ( $\gamma=1$ and $\beta=0.5$, respectively).

The discussion of Ref. [16] assumes that the elongation of critical fluctuations along the direction of $E$ can become so strong that fluctuations in the direction perpendicular to $E$ may crossover from Ising to mean-field behaviors. The crossover condition can be expressed as: $\xi_{\|}-\xi_{\perp} \approx \xi$, where $\xi$ is the correlation range in absence of $E$, and $\xi_{\|}$and $\xi_{\perp}$ are, respectively, the correlation ranges parallel and perpendicular to $E$. Taking into account

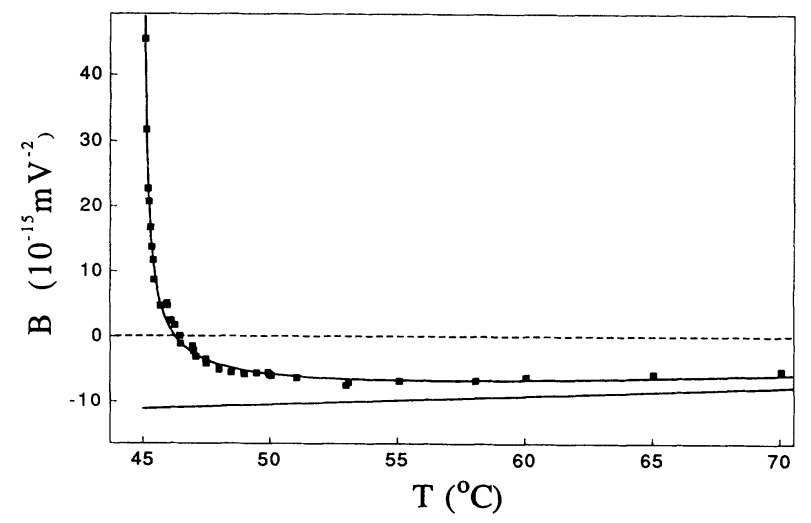

FIG. 2. The Kerr constant of the liquid mixture 2phenylethanol and decane, measured as a function of the temperature. Continuous lines show the noncritical background contribution and the best-fitting curve describing the superposition of critical and background contribution. 


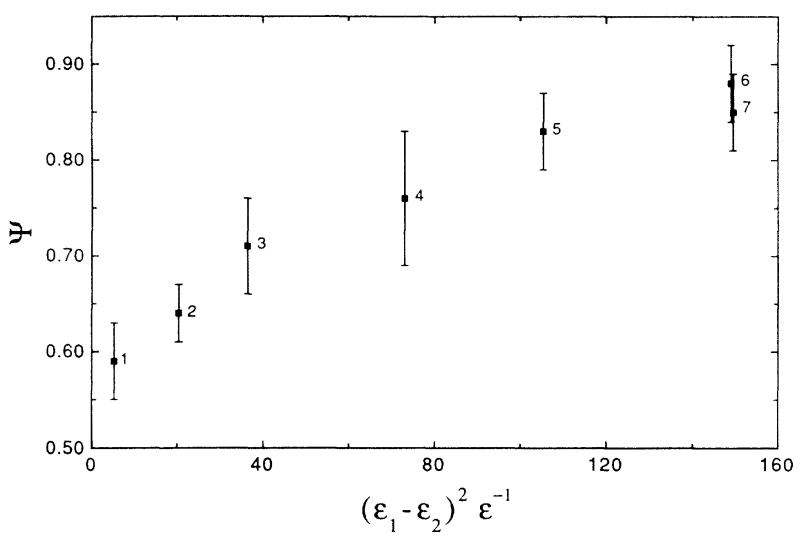

FIG. 3. The measured critical exponent $\psi_{\text {EKE }}$ plotted as a function of the quantity $\left(\varepsilon_{1}-\varepsilon_{2}\right)^{2} / \varepsilon$ for seven critical solutions: (1) ( \pm )-1-phenylethanol-dodecane; (2) 2-phenylethanoldodecane; (3) phenylacetonitrile-cyclopentane; (4) nitrobenzene-hexane; (5) propylene carbonate- $n$-butylbenzene; (6) lutidine-water; and (7) butoxyethanol-water.

that, at a given temperature, the size of fluctuations follows a probability distribution, and that the induced shape anisotropy grows with the size of fluctuations, the model of Ref. [16] assumes that the effective critical exponent $\psi_{\mathrm{EKE}}$ is determined by the sum of two contributions; the first, due to small fluctuations, which is described by the nonclassical exponent $\psi_{\mathrm{EKE}}=0.59$; and the second, due to fluctuations large enough to present the crossover to mean-field behavior, which is described by the exponent $\psi_{\mathrm{EKE}}=0.85$. The actual value of $\psi_{\mathrm{EKE}}$ would therefore be determined by the relative weight of the two families of fluctuations, and should be an increasing function of the mean elongation of critical fluctuations.

In principle, if the model is correct, one should be able to see the crossover from $\psi_{\mathrm{EKE}}=0.59$ to 0.85 simply by increasing the value of $E$. In practice, there is a limited experimental window in which the measurements are feasible, because low values of $E$ do not give enough signal, and large values of $E$ may induce heating of the solution. In general, the degree of elongation of critical fluctuations in the field $E$ is proportional to the osmotic compressibility and to the excess of dielectric constant of fluctuations over the dielectric constant of the solution. Both factors depend markedly on the distance from the critical point. According to the theoretical models [13-15], the amplitude $A_{\mathrm{EKE}}$ is given by

$$
A_{\mathrm{EKE}} \approx \frac{\left(\varepsilon_{1}-\varepsilon_{2}\right)^{2}\left(n_{1}^{2}-n_{2}^{2}\right)^{2}}{\varepsilon n^{2}},
$$

where $n_{1}$ and $n_{2}$ are the indices of refraction of the two components, and $n$ that of the mixture. The amplitude $A_{\text {EKE }}$ is the product of two terms, the first $\left(\varepsilon_{1}-\varepsilon_{2}\right)^{2} / \varepsilon$ describing the strength of the electric forces which induce the deformation of the correlation volume, and the second $\left(n_{1}^{2}-n_{2}^{2}\right)^{2} / n^{2}$ the optical anisotropy due to the induced deformation. By considering the structure of Eq. (5), it appears to be easy to change the magnitude of the induced anisotropy, at fixed reduced temperature and fixed field, by acting on the quantity $\left(\varepsilon_{1}-\varepsilon_{2}\right)^{2} / \varepsilon$.

It is noteworthy that the value of $\psi_{\mathrm{EKE}}$ measured with ( \pm )-1-phenylethanol-dodecane is smaller than any previous measurement of $\psi_{\mathrm{EKE}}$, and to our knowledge represents the first measurement of $\psi_{\mathrm{EKE}}$ which is in agreement with the theoretical predictions. The fact that such a result is obtained with the binary mixture possessing the smallest value of the quantity $\left(\varepsilon_{1}-\varepsilon_{2}\right)^{2} / \varepsilon$ is consistent with the hypothesis discussed in Ref. [16].

In Fig. 3 we have plotted the measured $\psi_{\mathrm{EKE}}$ as a function of $\left(\varepsilon_{1}-\varepsilon_{2}\right)^{2} / \varepsilon$ for the three systems studied in this work. We have also added data taken from Refs. [4], [6], and [7]. Figure 3 shows that a correlation exists between the value of $\left(\varepsilon_{1}-\varepsilon_{2}\right)^{2} / \varepsilon$ and the measured critical exponent $\psi_{\mathrm{EKE}}$. For mixtures presenting large values of $\left(\varepsilon_{1}-\varepsilon_{2}\right)^{2} / \varepsilon$, such as water-lutidine [6], waterbutoxyethanol [7] and nonionic micellar solutions [5(b)], the measured $\psi_{\text {EKE }}$ agrees, within experimental errors, with the asymptotic value 0.85 predicted in Ref. [16].

As a conclusion, we have shown in this paper, by using the present accurate data and also some literature data, that the effective critical exponent for the divergence of the Kerr constant in a critical binary mixture is a monotonically increasing function of the mismatch in the dielectric constant of the two components. When the mismatch is small, the data are in agreement with the "Ising" exponent predicted by theory. When the mismatch is large, the measured critical exponent takes a value consistent with the hypothesis that the fieldinduced anisotropy may become so strong to cause a crossover from Ising to mean-field behaviors.

\section{ACKNOWLEDGMENTS}

We thank T. Bellini, R. Piazza, and W. Pyzuk for useful discussions. We acknowledge financial support from the Italian Ministry for University and Research (MURST 40\% funds). One of the authors (S.J.R.) was supported by a grant from the International Center for Theoretical Physics, Trieste, Italy, under the Training Programme in Italian Laboratories.
[1] W. Pyzuk and H. Majgier-Baranowska, Chem. Phys. Lett. 63, 184 (1979).

[2] W. Pyzuk, H. Majgier-Baranowska, and J. Ziolo, Chem. Phys. 59, 111 (1980).

[3] W. Pyzuk, Chem. Phys. 50, 281 (1980).
[4] W. Pyzuk, Europhys. Lett. 17, 339 (1992).

[5] V. Degiorgio and R. Piazza, (a) Phys. Rev. Lett. 55, 288 (1985); (b) Prog. Colloid. Polymer, Sci. 73, 76 (1987).

[6] R. Piazza, T. Bellini, V. Degiorgio, R. E. Goldstein, S. Leibler, and R. Lipovsky, Phys. Rev. B 38, 7223 (1988). 
[7] T. Bellini and V. Degiorgio, Phys. Rev. B 39, 7263 (1989).

[8] S. J. Rzoska, V. Degiorgio, T. Bellini, and R. Piazza, Phys. Rev. E 49, 3093 (1994).

[9] D. Wirtz, K. Berend, and G. G. Fuller, Macromolecules 25, 7234 (1992).

[10] S. J. Rzoska, J. Chrapec and J. Ziolo, Physica A 139, 569 (1986).

[11] S. J. Rzoska, Phase Trans. 27, 1 (1990).

[12] S. J. Rzoska and J. Ziolo, Phys. Rev. E 47, 1445 (1993).

[13] J. Goulon, J. L. Greffe, and D. W. Oxtoby, J. Chem. Phys. 70, 4742 (1979).
[14] J. S. Høye and G. Stell, J. Chem. Phys. 81, 3200 (1984).

[15] A. Onuki and M. Doi, Europhys. Lett. 17, 63 (1992).

[16] S. J. Rzoska, Phys. Rev. E 48, 1136 (1993).

[17] E. Fredericq and C. Houssier, Electric Dichroism and Electric Birefringence (Clarendon, Oxford, 1973).

[18] J. Ziolo, S. J. Rzoska, and J. Chrapec, Phase Trans. 9, 317 (1987).

[19] B. Scaife, Principles of Dielectrics (Clarendon, Oxford, 1989).

[20] D. Beysens, R. Gaustaud, and F. Decruppe, Phys. Rev. A 30, 1145 (1984). 